\title{
تحديد مقاومة عزلة محلية للبكتريا المحبة للحرارة Bacillus stearothermophilus و المعزولة من التربة لبعض إضير العناصر الثقيلة وتأثيراتها الضارة
}

\section{Determination of Heavy Metals Tolerance and their Damaging Effects on a Local Soil Thermophilic Isolate Bacillus stearothermophilus}

\author{
خلود عبد الاله محمد الخفاجي مائرة الزر اعية/ وزارة العلوم والتكنولوجيا صفاء عبد الرحيم محمود \\ Khlood A. A. Al-Khafaji Saffa A. Raheem
}

Agricultural Research Directorate/ Ministry of Science and Technology

E-mail: khloodalkhafaji@yahoo.com

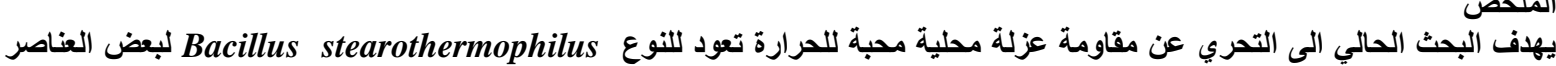

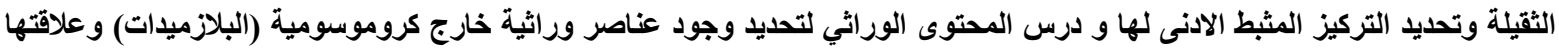

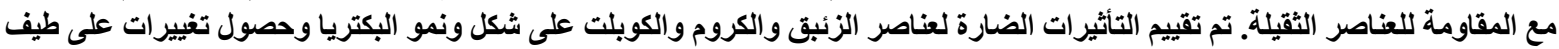

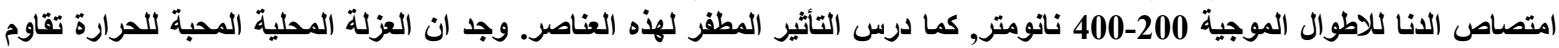

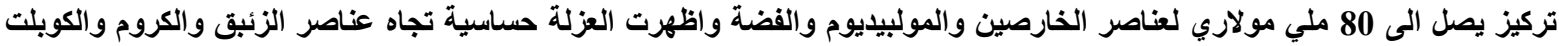

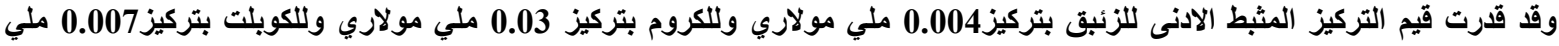

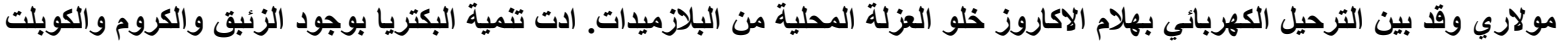

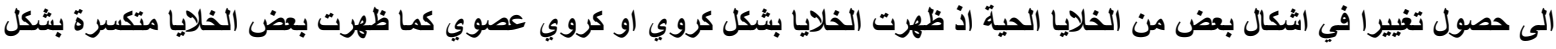

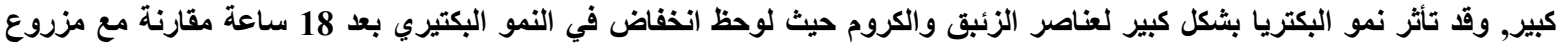

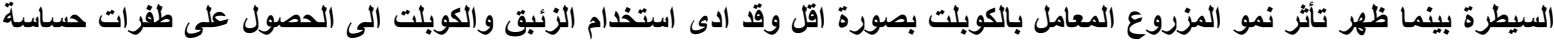

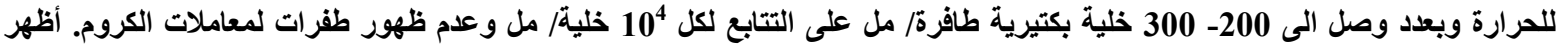

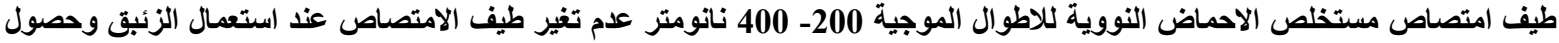

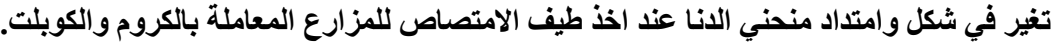

الكلمات المفتاحية: بكتريا محبة للحرارة, B. stearothermophilus, عناصر ثقيلة, بلازميدات, تركيز مثبط ادنى, تطفير, طيف امتصاص، الاحماض النووية.

Abstract

This research aimed to detect the tolerance of a local soil thermophilic isolate to some heavy metals (HM) and determine of minimal inhibition concentration (MIC). Also, plasmid profile was studied and its relationship with HM resistant. Deleterious effect of mercury $(\mathrm{Hg})$, chromium $(\mathrm{Cr})$ and Cobalt $(\mathrm{Co})$ were evaluated on bacterial shape, growth and DNA scan between 200-40nm. Mutation effect was also studied. Local thermophilic Bacillus stearothermophilus was resist zinc, molbidium, silver at a concentration reached $80 \mathrm{mM}$; it is sensitive to $\mathrm{Hg}, \mathrm{Cr}$ and $\mathrm{Co}$ with $\mathrm{MIC}$ reached $0.004,0.03$ and 0.007 respectively. Plasmid profile revealed the absence of plasmid from B. stearothermophilus. Some of bacterial shapes were changed to short bacilli, coccobacilli and cocci; many bacterial cell fragment were seen after treatment with $\mathrm{Hg}, \mathrm{Cr}$ and $\mathrm{Co}$. Bacterial growth decreased when treated with $\mathrm{Hg}$ and $\mathrm{Cr}$ after 18h while, little effect was seen with Co treatment with $\mathrm{Hg}$ and $\mathrm{Co}$ induced heat sensitive mutation with 200-300 mutated $\mathrm{cfu} / \mathrm{ml}$ from $10^{4} \mathrm{cfu} / \mathrm{ml}$; no heat sensitive mutation were detected for $\mathrm{Cr}$ treatment. DNA UV scan 200-400nm showed no changes with $\mathrm{Hg}$ while, curve shape changed for $\mathrm{Cr}$ and cobalt.

Key words: thermophilic bacteria, Bacillus stearothermophilic, heavy metal, plasmid, MIC, mutation

المقدمة

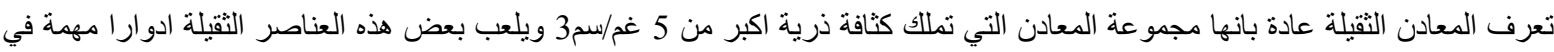

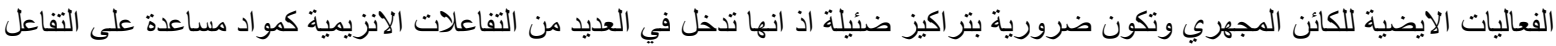

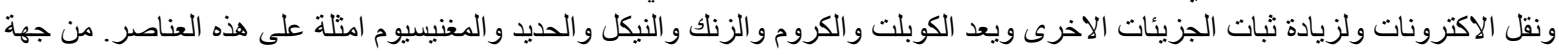

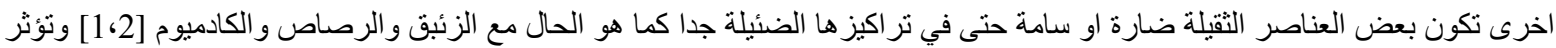

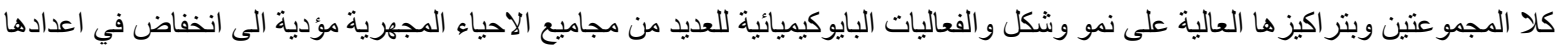

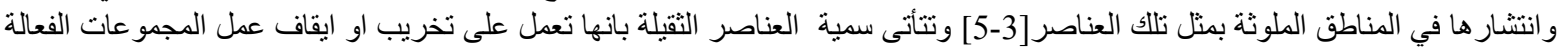

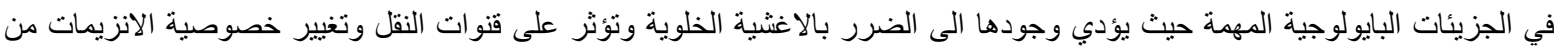

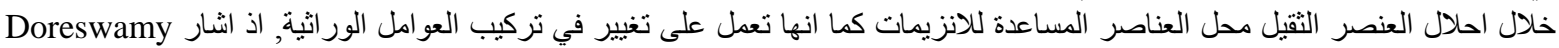

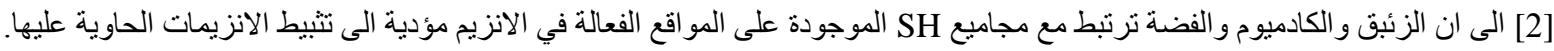

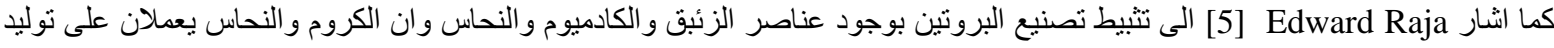


مجاميع اوكسجين فعالة والتي تعمل على تحفيز اضافة مجاميع البيروكسيد لدهون الاغشية الخلوية وحصول الضرر التأكسدي. وتعتبر الاحياء

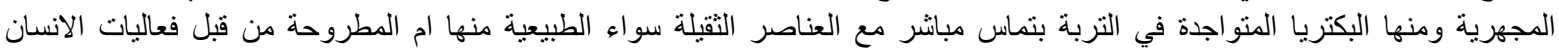

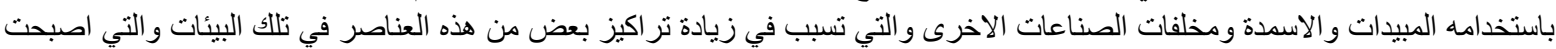

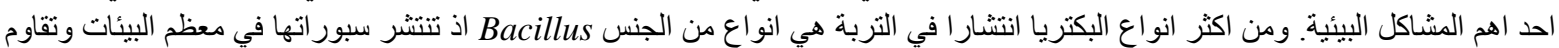

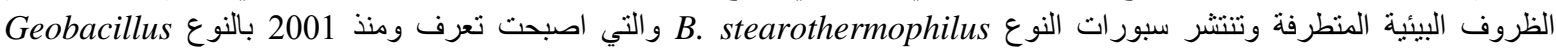
stearothermophilus

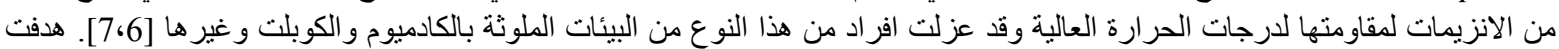

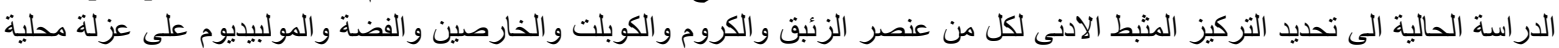

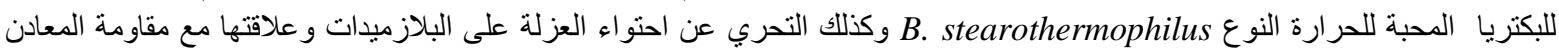

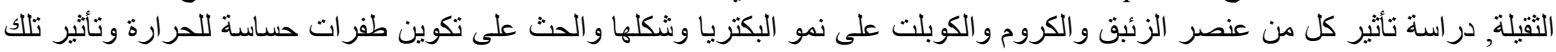

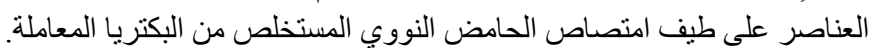

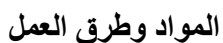

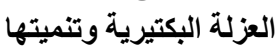

نم اعتماد العزلة البكتيرية المحبة للحر ارة B. stearothermophilus و التي عزلت وشئ وشخصت من قبل الخفاجي [8]. نميت العزلات البكتيرية

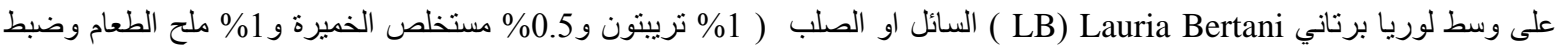

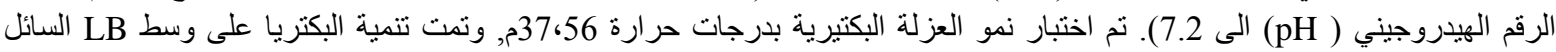

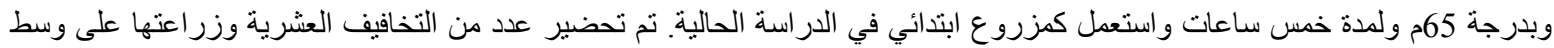
LB

تحضير محاليل المعادن الثقيلة

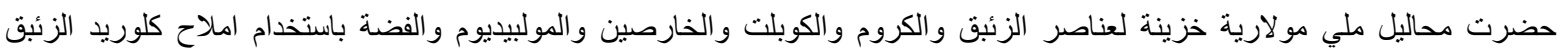

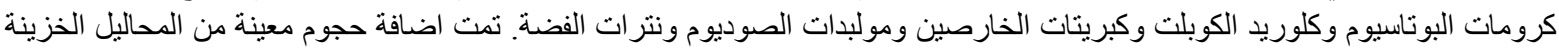

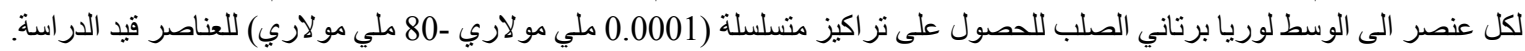

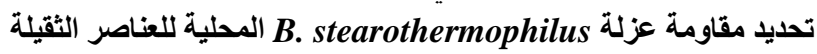

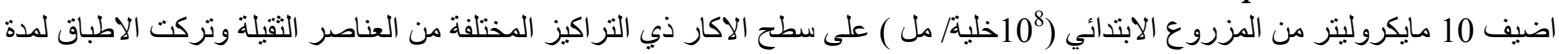

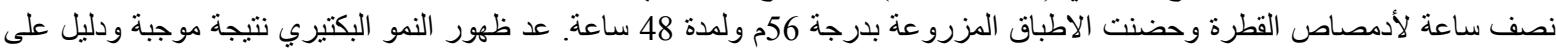

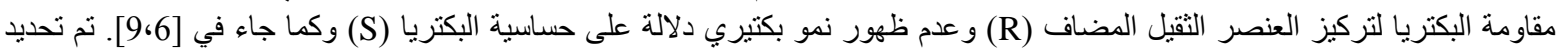

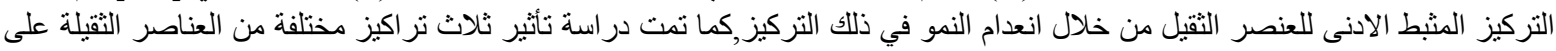

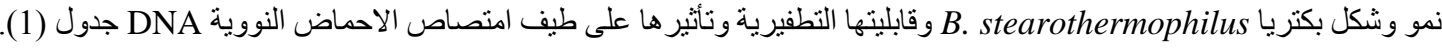

\begin{tabular}{|c|c|c|c|}
\hline \multicolumn{4}{|c|}{ 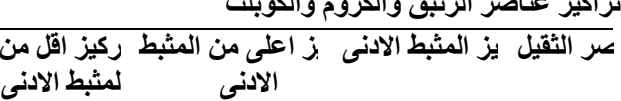 } \\
\hline 0.002 & 0.005 & 0.004 & الزئبق \\
\hline 0.02 & 0.05 & 0.03 & 'لكروم \\
\hline 0.005 & 0.01 & 0.007 & كوبلت \\
\hline
\end{tabular}

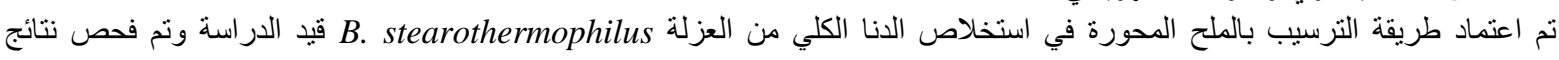

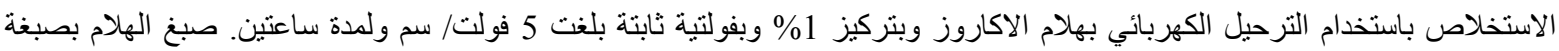

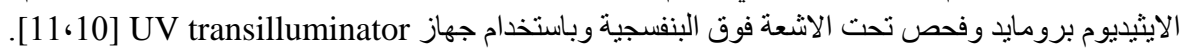

B. stearothermophilus تأثير العناصر الثقيلة على نمو وشكل البكترين

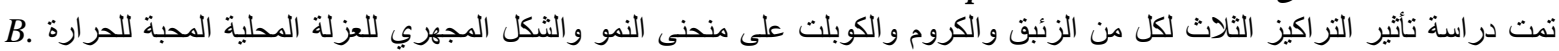

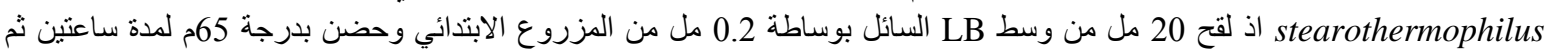

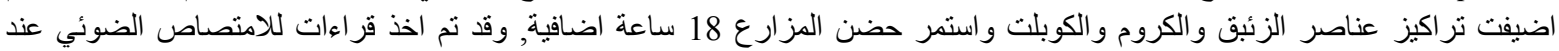

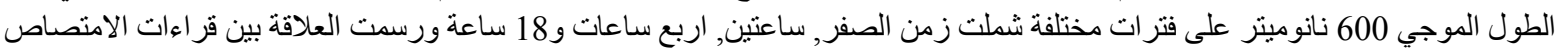

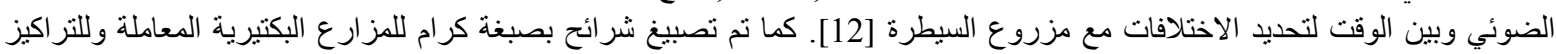

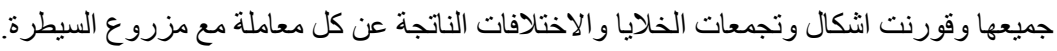

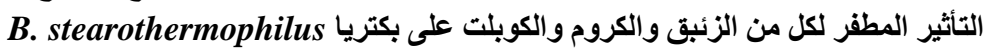

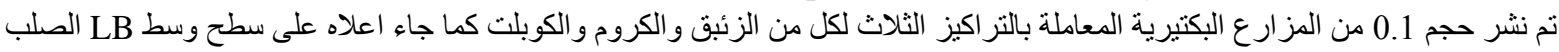

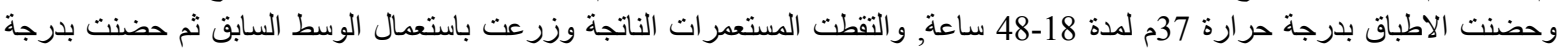

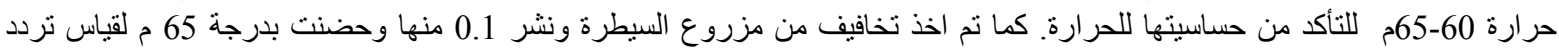

الطفر ات الحساسة للحر ارة [13] 
تأثير الزئبق والكروم والكوبلت على طيف امتصاص الاحماض النووية للاطوال 200- 400 نانوميتر

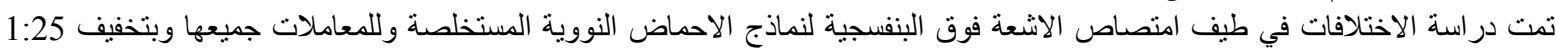
حيث قرأ طيف امتصاص الاشعة فوق البنفجية بين الطول الموجي 200 اللى 400 نانوميتر لكل نموذج وباستعمال جهاز المطياف الضونئي . اعتمادا على [14] (Analytikjena specord 205, Germany) النتائج و المناقشة

اعتمدت العزلة المحلية المحبة للحر ارة والتي تعود للنوع البكتيري B. stearothermophilus في تحديد التر اكيز المثبطة الدنيا لكل من الزئبق

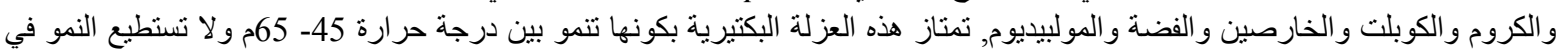

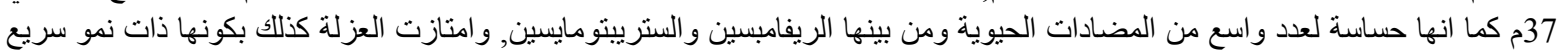

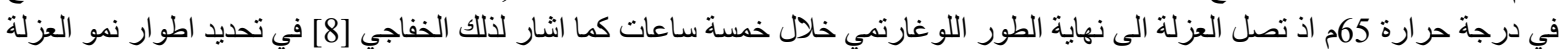

المحلية.

أوضح فحص مقاومة العزلة المحلية لبكتريا B. stearothermophilus لعدد من التر اكيز المتسلسلة لعناصر شملت الزئبق و الكروم و الكوبلت

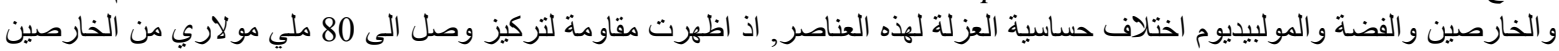

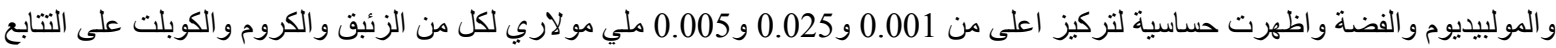

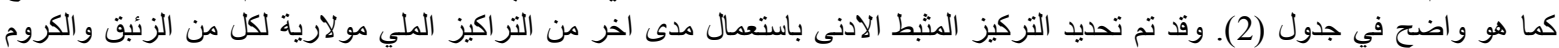
و الكوبلت لتكون التر اكيز المثبطة الدنيا

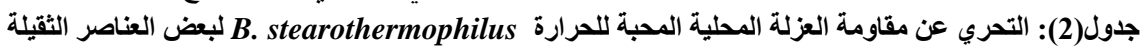

\begin{tabular}{|c|c|c|c|c|c|c|c|c|c|c|c|c|c|c|c|c|}
\hline & \multicolumn{15}{|c|}{ تركيز العنصر الثقيل (ملي مولاري) } & العنصر \\
\hline 80 & 40 & 20 & 10 & 5 & 1.6 & 0.8 & 0.4 & 0.2 & 0.1 & 0.05 & 0.025 & 0.01 & 0.005 & 0.001 & 0.0005 & 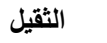 \\
\hline $\mathbf{S}$ & $\mathbf{S}$ & $\mathbf{S}$ & $\mathbf{S}$ & $\mathbf{S}$ & $\mathbf{S}$ & $\mathbf{S}$ & $\mathbf{S}$ & $\mathbf{S}$ & $\mathbf{S}$ & $\mathbf{S}$ & $\mathbf{S}$ & $\mathbf{S}$ & $\mathbf{S}$ & $\mathbf{R}$ & $\mathbf{R}$ & 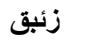 \\
\hline $\mathbf{S}$ & $\mathbf{S}$ & $\mathbf{S}$ & $\mathbf{S}$ & $\mathbf{S}$ & $\mathbf{S}$ & $\mathbf{S}$ & $\mathbf{S}$ & $\mathbf{S}$ & $\mathbf{S}$ & $\mathbf{S}$ & $\mathbf{R}$ & $\mathbf{R}$ & $\mathbf{R}$ & $\mathbf{R}$ & $\mathbf{R}$ & كروم \\
\hline $\mathbf{S}$ & $\mathbf{S}$ & $\mathbf{S}$ & $\mathbf{S}$ & $\mathbf{S}$ & $\mathbf{S}$ & $\mathbf{S}$ & $\mathbf{S}$ & $\mathbf{S}$ & $\mathbf{S}$ & $\mathbf{S}$ & $\mathbf{S}$ & $\mathbf{S}$ & $\mathbf{R}$ & $\mathbf{R}$ & $\mathbf{R}$ & كوبلت \\
\hline $\mathbf{R}$ & $\mathbf{R}$ & $\mathbf{R}$ & $\mathbf{R}$ & $\mathbf{R}$ & $\mathbf{R}$ & $\mathbf{R}$ & $\mathbf{R}$ & $\mathbf{R}$ & $\mathbf{R}$ & $\mathbf{R}$ & $\mathbf{R}$ & $\mathbf{R}$ & $\mathbf{R}$ & $\mathbf{R}$ & $\mathbf{R}$ & مولبيديوم \\
\hline $\mathbf{R}$ & $\mathbf{R}$ & $\mathbf{R}$ & $\mathbf{R}$ & $\mathbf{R}$ & $\mathbf{R}$ & $\mathbf{R}$ & $\mathbf{R}$ & $\mathbf{R}$ & $\mathbf{R}$ & $\mathbf{R}$ & $\mathbf{R}$ & $\mathbf{R}$ & $\mathbf{R}$ & $\mathbf{R}$ & $\mathbf{R}$ & خارصين \\
\hline $\mathbf{R}$ & $\mathbf{R}$ & $\mathbf{R}$ & $\mathbf{R}$ & $\mathbf{R}$ & $\mathbf{R}$ & $\mathbf{R}$ & $\mathbf{R}$ & $\mathbf{R}$ & $\mathbf{R}$ & $\mathbf{R}$ & $\mathbf{R}$ & $\mathbf{R}$ & $\mathbf{R}$ & $\mathbf{R}$ & $\mathbf{R}$ & فضة \\
\hline
\end{tabular}

قد نطور الاحياء المجهرية واحدة او اكثر من اليات مقاومة العناصر الثقيلة السامة لتمكنها من مقاومة التراكيز العالية والمتوفرة في بيئاتها ومن

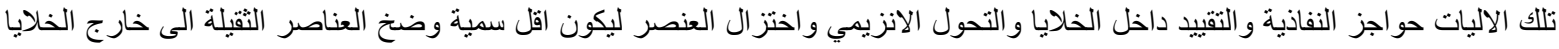

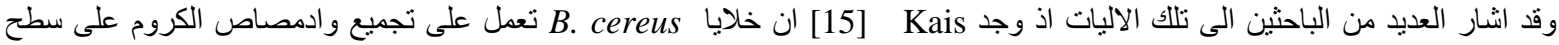

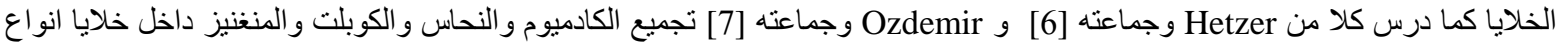

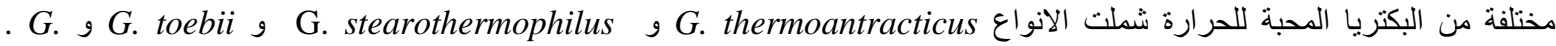
بكتريا thermoleovorans

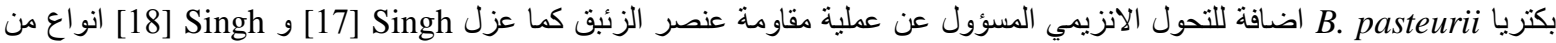

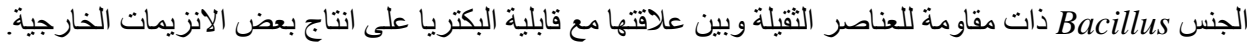

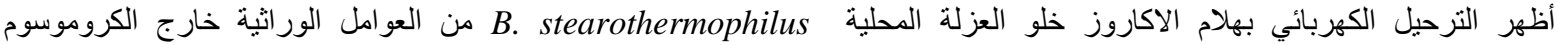

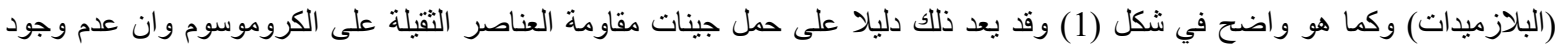

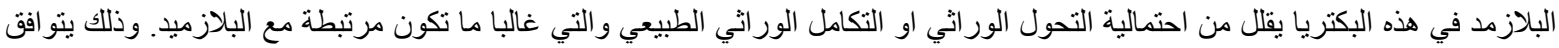

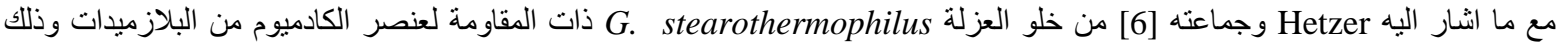

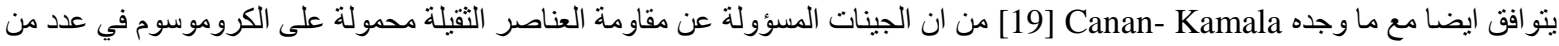
عز لات Bacillus اذ ان تحييد البلازميدات في هذه العزلات لم تؤثر على مقاومتها للعناصر الثقيلة والعديد من المضادات الحيوية بينما ادى تحييد

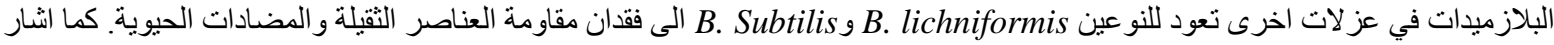

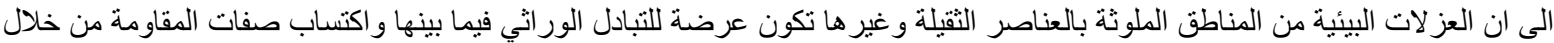




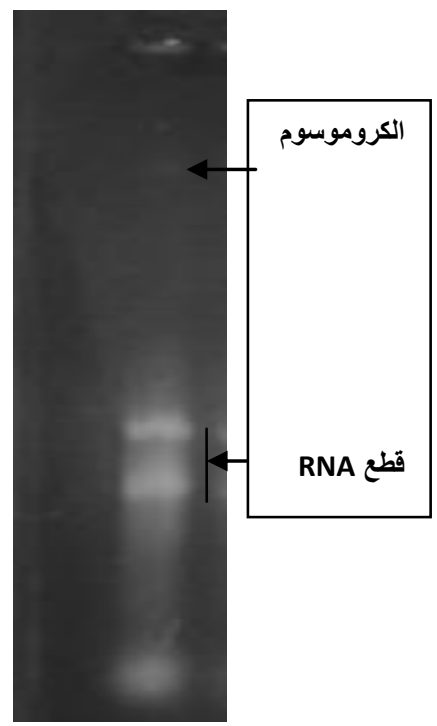

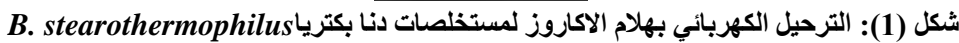

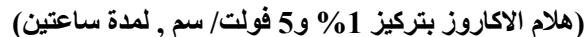

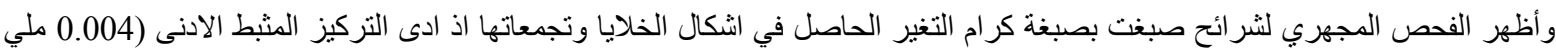

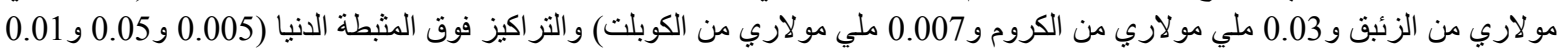

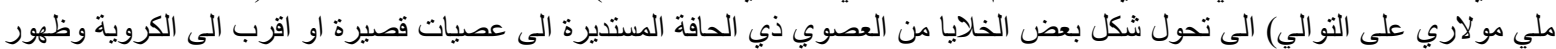

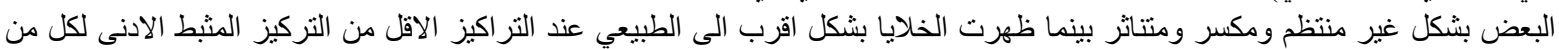

الزئبق و الكروم و الكوبلت وبتر اكيز ملي مو لارية شملت 0.002 و 0.02 و 0.005 على التتابع شكل (2).

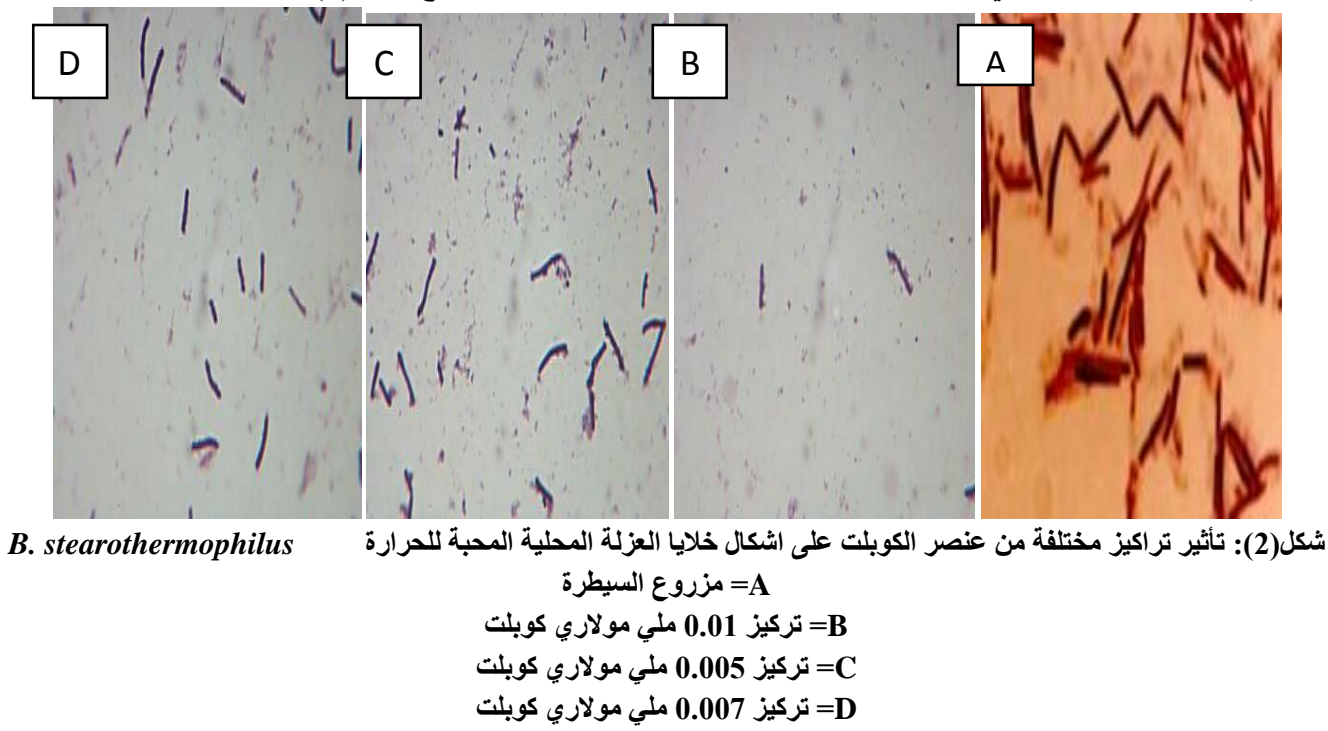

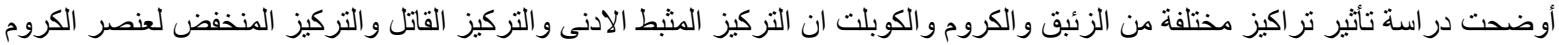

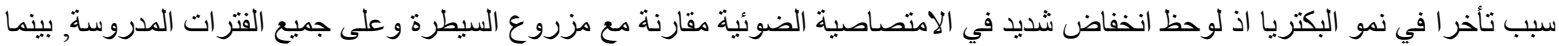

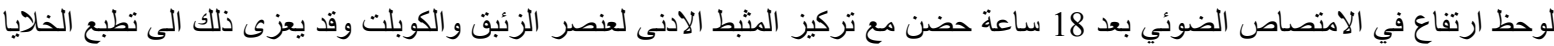

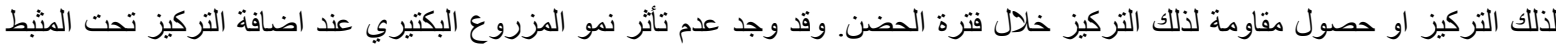

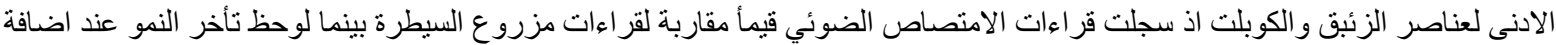

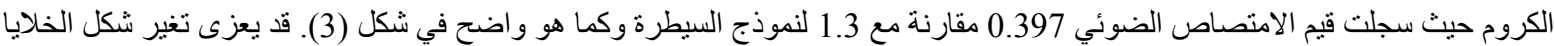

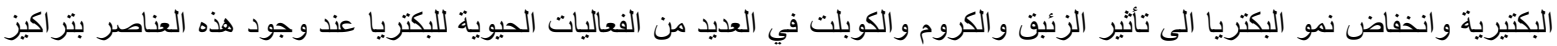

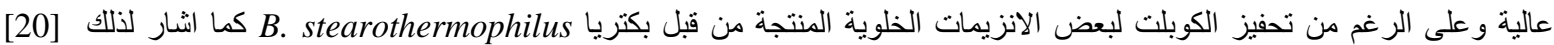
Markowicz

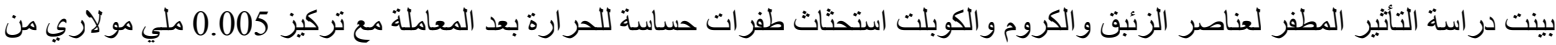

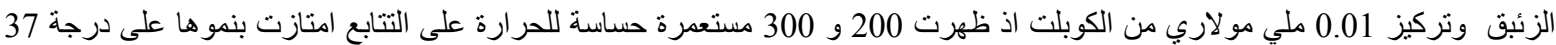

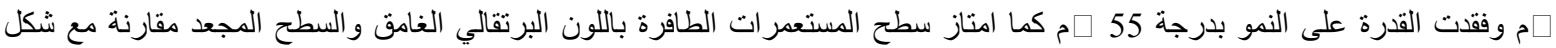
المستعمرة الام ذات الثكل الثفاف و السطح الاملس. 


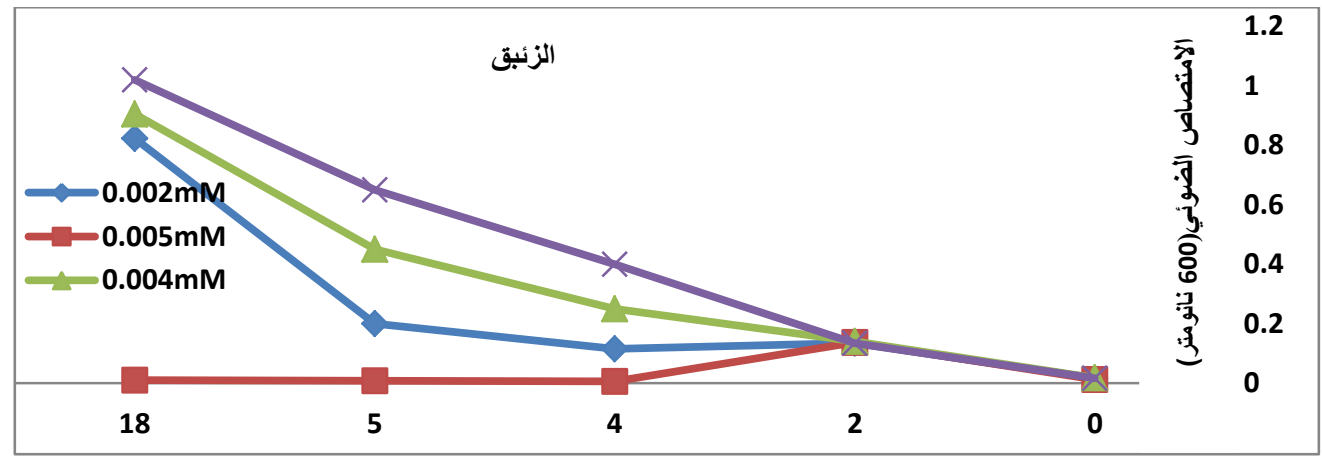

الو قت (ساعة)

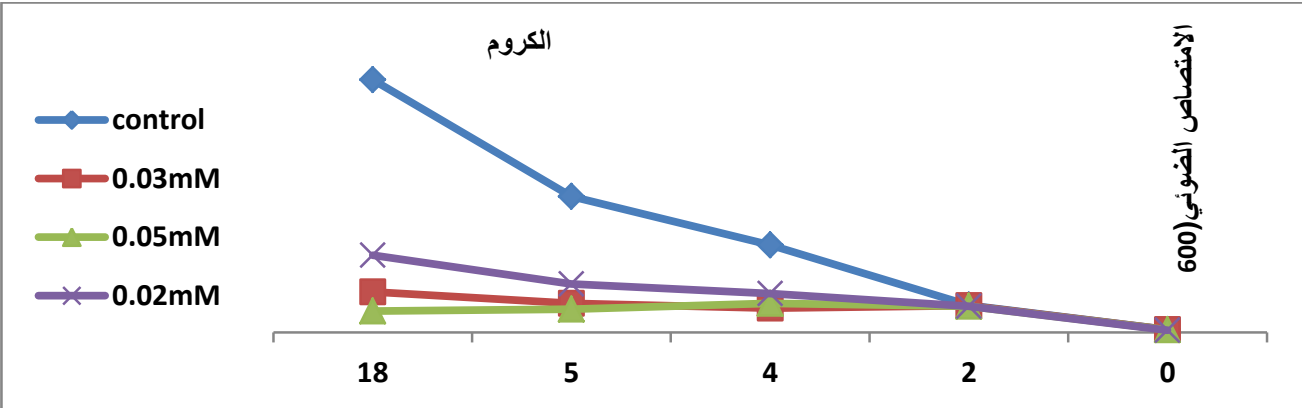

الوقت (ساعة)

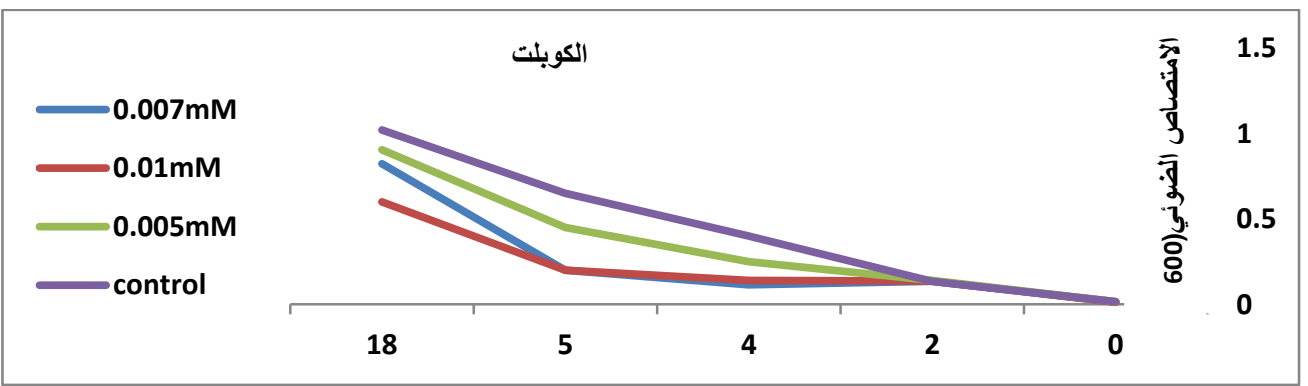

الوقت (ساعة)

B. stearothermophilus شكل(3): تأثير تراكيز مختلفة من العناصر الثقيلة على نمو العزلة المحلية المحبة للحرارة

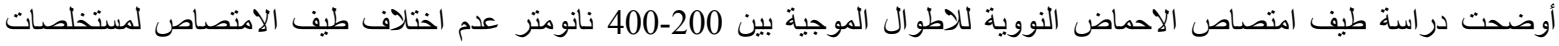

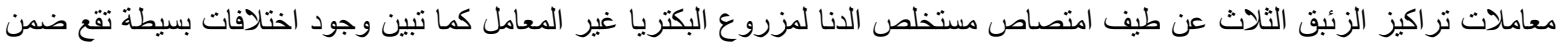

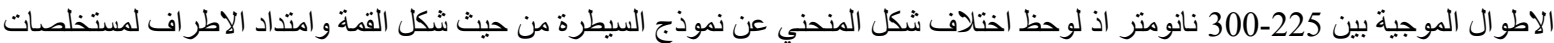

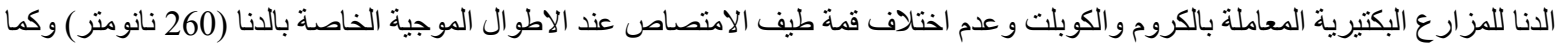

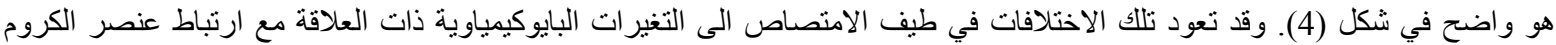
او الكوبلت وتأثير اتهما وتغير المحتوى الور اثي الخلوي. 

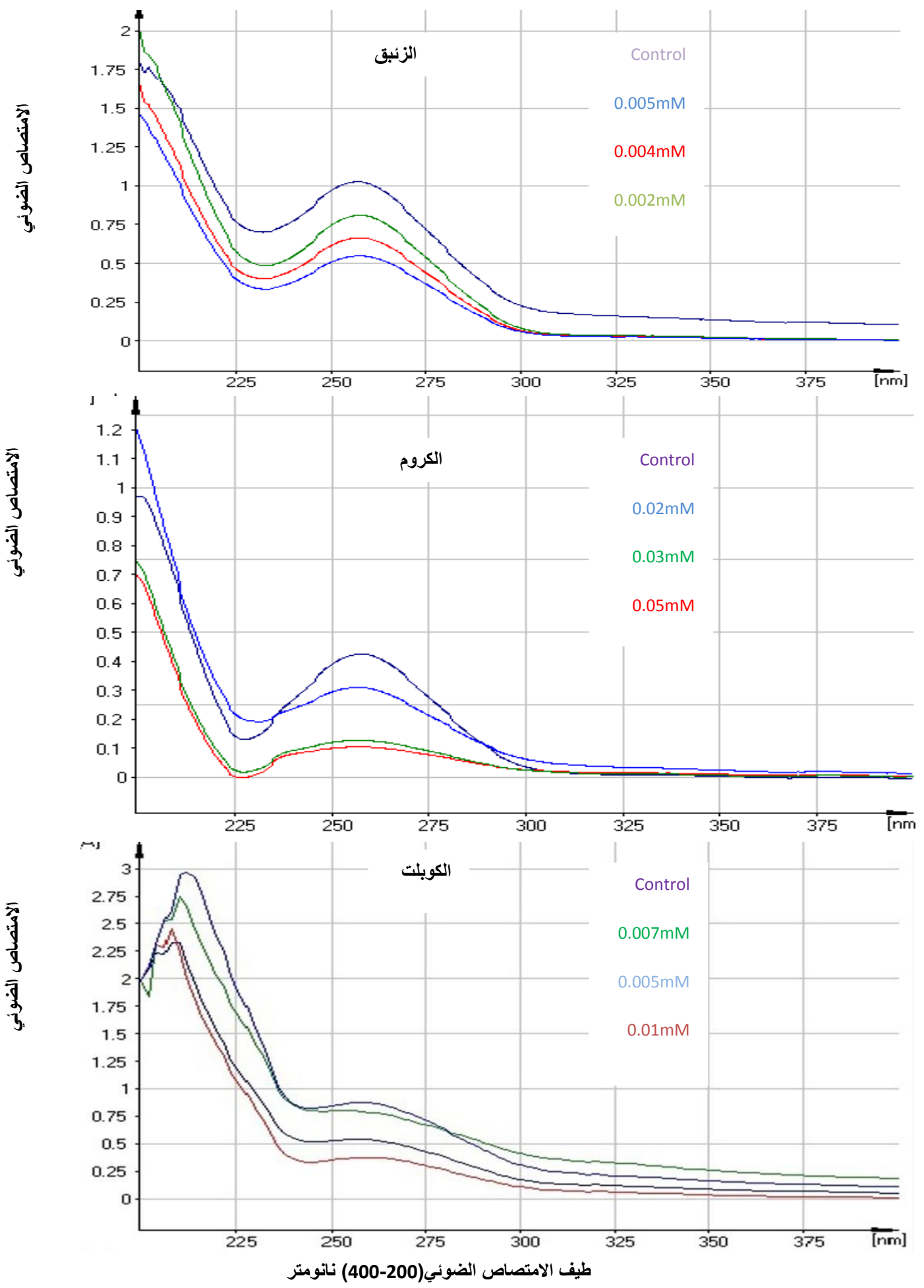

شكل(4): طيف امتصاص الاحماض النووية عند الاطوال الموجية 200-400 نانوميتر للعزلة المحلية مقام B. stearothermophilus

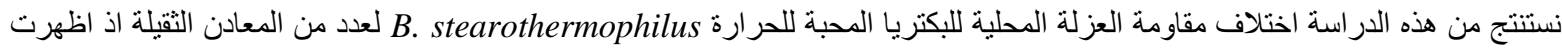

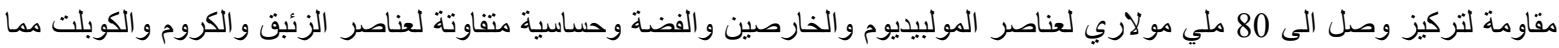

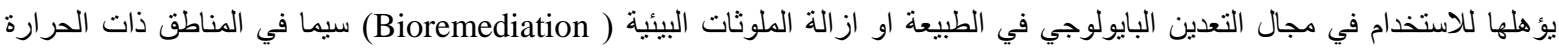

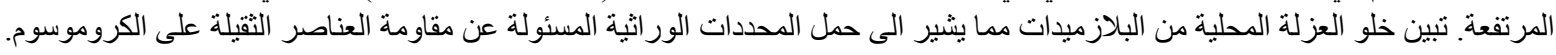

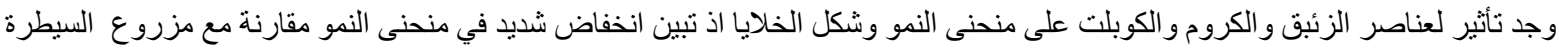

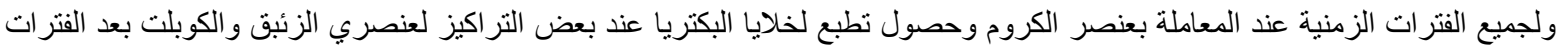

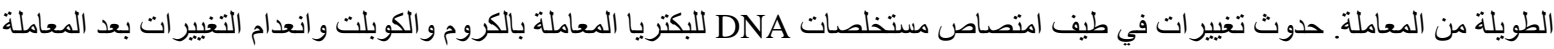
بالزئبق مع وجود نأثثر مطفر لعنصر الزئبق في تر اكيزه العالية. 


\section{References}

1. Brunis, M. R. (2000). Microbial resistance of metals in the environment. Ecotoxicol. Environm. Saf. 45:198206.

2. Doreswamy, K. (2004). Nickel induced oxidative stress in testis of mice. Evidence of DNA damage and genotoxic effects. J. Androil, 25:996.

3. Hassen, A. (1998). Effects of heavy metals on Pseudomonas aeruginosa and Bacillus thuringensis. Bioresource. Technol65:73.

4. Nies, D. H. (1999). Microbial heavy metal resistant. Applied Microbiology Biotechnology. 51: 730- 750.

5. Edward Raja, C., Selvam, G. S. and Omine, K. (2009). Isolation, identification and characterization of heavy metal resistant bacteria from sewage. International joint Symposium on Geodisaster Prevention and Geoenvironment in Asia. 205- 211.

6. Hetzer, A., Dauphney, C. J., and Morgan, H. W. (2006). Cadmium ion biosorption by the thermophilic bacteria Geobacillus stearothermophilus and G.thermocatenulatus. Appl. Environ. Microbiol. 72(6): 4020- 4027.

7. Ozdemir, S., Kilinc, E., Po, A., Nicolans, B. and Guven, K. (2012). Cd, Cu, Mn and Zn resistance and bioaccumulation by thermophilic bacteria, Geobacillus toebii subsp decanicus and Geobacillus thermoleovorans subsp. stromboliensis. World J. of Microbiol. And Biotechnol. 28(1): 155- 163.

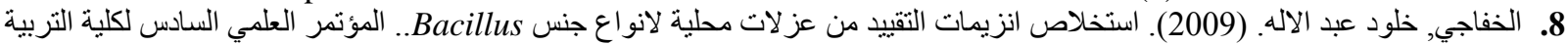
/جامعة تكريت/ قسم علوم الحياة. 24-25 الاله

9. Filali, B. K., Taoufik, J., Zeroual, Y., Dzairi, F. Z., Talbi, M. and Blaghen. (2000). Waste water bacterial isolates resistant to heavy metals and antibiotics. Current Microbiology. 41: 151- 156.

10. Al-khafaji, K. A. and Al- Thwani, A.N. (2011). A comparative study among clinical and environmental isolates of Vibrio cholerae at their antibiotic resistance and plasmid profile. J. biotechnology research center(special ed.). vol.5 (2):3-9

11. Sambrook, J., Fritsh, F. E. and Maniatis , T. (1989). Molecular cloning: A laboratory manual 2nd Ed . Cold spring Harbor Laboratory press. New York.

12. Rathnayake, I. V. N., Megharaj, M., Bolan, N. and Naidu, R. (2009). Tolerance of heavy metals by gram positive soil bacteria. World Academy of Science and Technology. 53: 1185- 1189.

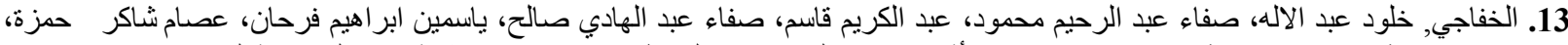

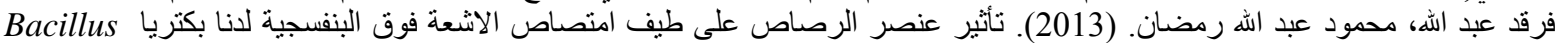
stearothermophilus

14. Morys, M. and Daniel, B. (1993). The accurate measurements of biologically effective ultraviolet radiation. International Symposium on High Latitude Optics, Tromso, Norway.

15. Kais, K. G., Al- Daraghi, W. A. and Lateef, N. S. (2013). Study the heavy metals tolerance biosorption and antibiotic resistance of Bacillus cereus isolated from diesel fuel polluted soil. International J. Biological and Pharmaceutical Research. 4(7): 502-506.

16. Pahan, K., Chaudhuri, J., Ghosh, D.K., Gachhui, R., Ray, S. Mandal, A. (1996). Voltalization of mercury from natural water by a broad- spectrum Hg- resistant Bacillus pasteurii strain DR2. The Environmentalist. 16(3): 179- 185.

17. Singh, S. K., Tripathi, V. R., Jain, R. K., Vikram, S. and Garg, S. K. (2010). An antibiotic, heavy metal resistant and halotolerant Bacillus cereus SIU1 and its thermoalkaline protease. Microbial cell factory. 9:59.

18. Singh, Y., Ramteke, P. W., tripathy, A. and Shukla, P. K. (2013). Isolation and characterization of Bacillus resistant to multiple heavy metals. International J. Current Microbiology and Applied Sciences. 2(11):525530.

19. Kamala- Kannan, S. and Lee, K. J. (2008). Metal tolerance and antibiotic resistance of Bacillus isolated from Sunchon Bay sediments, South Korea. Biotechnology. 7(1): 149- 152.

20. Markowicz, A., Plociniczak, T. and Piotrowska- Seget, Z. (2010). Response of bacteria to heavy metals measured as changes in FAME profiles. Polish J. of Environ. Stud. 19(5): 957- 965. 